\title{
Neuropatía óptica bilateral e irreversible por ingestión de disolvente de pintura
}

\section{Bilateral and irreversible optic neuropathy due to ingestion of paint thinner}

\author{
Celia Ruiz-Arranz*, José A. Reche-Sainz, Sara E. Hernández-Almeida, Cristina Calleja-García y \\ Manuel Ferro-Osuna
}

Servicio de Oftalmología, Hospital Universitario 12 de Octubre, Madrid, España

\section{Resumen}

Objetivo: Describir las manifestaciones clínicas y la evolución de un paciente con intoxicación aguda por ingestión de disolvente de pintura. Observaciones: Varón de 35 años que fue atendido por un cuadro de agitación y desorientación. Había consumido por divertimento una mezcla de alcohol, diazepam y marihuana, y se había bebido una cantidad indeterminada de disolvente de pintura que contenía metanol y tolueno. Desarrolló una acidosis metabólica muy grave con anión gap aumentado e hipopotasemia que precisó medidas correctoras del equilibro hidroelectrolítico. La agudeza visual estaba muy disminuida bilateralmente hasta percepción de luz y presentaba edema de papila tenue que evolucionó a atrofia óptica total, sin mejoría. Conclusiones: En las intoxicaciones agudas por metanol y tolueno, ambos pueden producir síntomas psicoactivos y acidosis metabólica grave. La hipopotasemia con debilidad muscular es característica del tolueno, mientras que el metanol es la causa de neuropatías ópticas agudas bilaterales de carácter grave y progresivo.

Palabras clave: Metanol. Tolueno. Intoxicación aguda. Neuropatía óptica. Acidosis metabólica. Hipopotasemia.

\begin{abstract}
Objective: To describe the clinical manifestations and evolution of a patient with acute poisoning due to ingestion of paint thinner. Observations: A 35-year-old man who was treated for agitation and disorientation. He had consumed a mixture of alcohol, diazepam, and marijuana for fun, and had drunk an undetermined amount of paint thinner containing methanol and toluene. He developed a very severe metabolic acidosis with an increased anion gap and hypokalemia that required corrective measures of the fluid and electrolyte balance. Visual acuity was greatly decreased bilaterally to light perception, and he had mild papilledema that progressed to total optic atrophy, without visual improvement. Conclusions: In acute poisoning by methanol and toluene, both can produce psychoactive symptoms and severe metabolic acidosis. Hypokalemia with muscle weakness is characteristic of toluene, while methanol produces severe and progressive bilateral acute optic neuropathies.
\end{abstract}

Keywords: Methanol. Toluene. Acute poisoning. Optic neuropathy. Metabolic acidosis. Hypokalemia.

Fecha de recepción: 10-12-2020 Av. de Córdoba s/n, C.P.: 28041, Madrid, España E-mail: celia.ruiz.arranz@ hotmail.com DOI: 10.24875/RMO.M21000195
Fecha de aceptación: 06-04-2021
Disponible en internet: $25-10-2021$ Rev Mex Oftalmol. (ahead of print) www.rmo.com.mx 0187-4519/@ 2021 Sociedad Mexicana de Oftalmología. Publicado por Permanyer. Este es un artículo open access bajo la licencia CC BY-NC-ND (http://creativecommons.org/licenses/by-nc-nd/4.0/). 


\section{Introducción}

Los disolventes orgánicos son compuestos con una estructura básica de carbono e hidrógeno, con múltiples aplicaciones en las industrias siderúrgica, farmacéutica y cosmética, así como en la fabricación de plásticos, productos de limpieza, pinturas, lacas, adhesivos y pegamentos $^{1,2}$.

Generalmente son muy volátiles a temperatura ambiente, lo que facilita su entrada en el organismo por vía inhalatoria. Además, por su naturaleza lipófila, tienen una gran afinidad por los tejidos nerviosos y los recubrimientos de mielina, produciendo efectos neurotóxicos ${ }^{1-4}$.

La exposición ocupacional se suele producir de forma crónica, habitualmente a lo largo un tiempo prolongado (años) y a bajas dosis ${ }^{1,2,4}$. Sin embargo, entre los adolescentes y la población joven no es infrecuente la inhalación intencionada de pegamentos o disolventes de pintura con fines recreativos ${ }^{3,5}$.

Se expone un caso de intoxicación aguda por inhalación e ingestión de disolvente de pintura.

\section{Presentación del caso}

Varón de 35 años que es traído a urgencias por un cuadro de desorientación y agitación. Había consumido por divertimento una mezcla de diazepam y alcohol, a la vez que fumaba marihuana e inhalaba disolvente de pintura, que terminó ingiriendo (cantidad no conocida). Inicialmente se encontraba agitado, poco colaborador y con bajo nivel de consciencia (8 puntos en la escala de Glasgow). Se objetivó una midriasis bilateral media arreactiva y en el fondo de ojo preservación de la coloración y de la microcirculación de ambas papilas, si bien había un borramiento poco marcado sin apenas elevación de los bordes (Fig. 1 A y B). La resonancia magnética cerebral no mostró hallazgos significativos. Se comprobó que la composición del disolvente de pintura ingerido era 50\% tolueno, 20\% metanol, $20 \%$ acetato de metilo y $10 \%$ propanona (acetona).

En la analítica sanguínea presentaba potasio $1.93 \mathrm{mEq} / \mathrm{l}$, cloro $93 \mathrm{mEq} / \mathrm{l}$ y etanol $<0.1 \mathrm{~g} / \mathrm{l}$, y en la gasometría $\mathrm{pH} 7.23, \mathrm{pCO}_{2} 17 \mathrm{mmHg}, \mathrm{HCO}_{3}^{-} 7 \mathrm{mmol} / \mathrm{l}$, anión gap 28 y lactato $1.2 \mathrm{mmol} / \mathrm{l}$. Fue ingresado con el diagnóstico de acidosis metabólica hipoclorémica con anión gap aumentado, hipopotasemia grave y neuropatía óptica tóxica bilateral, en el contexto de una intoxicación por metanol y tolueno. La detección de tóxicos en orina fue negativa, exceptuando benzodiacepinas, y en el electrocardiograma se objetivó un QT corregido largo (550 ms) con descenso del segmento ST. EI paciente fue tratado con clorazepato dipotásico (10 mg/12 h), bicarbonato, piridoxina (300 mg/24 h), tiamina $(300 \mathrm{mg} / 24 \mathrm{~h})$, folinato cálcico $(30 \mathrm{mg} / 12 \mathrm{~h})$, ácido folínico (50 mg/6 h) e infusión de etanol $(2 \mathrm{ml} / \mathrm{kg}$ al día), con lo que se corrigieron las alteraciones analíticas y electrocardiográficas durante los primeros 3 días de ingreso. Se le sometió a seis sesiones de hemodiálisis con el objetivo de eliminar los metabolitos tóxicos del metanol.

A los 3 días de evolución, la agudeza visual (AV) era de dudosa percepción de luz en el ojo derecho (OD) y de percepción y proyección de luz en el ojo izquierdo (OS), persistiendo un tenue edema papilar. Las pupilas estaban midriáticas, escasamente reactivas, con un defecto pupilar aferente en el OD. La tomografía de coherencia óptica (OCT) mostró un aumento del grosor de la capa de fibras nerviosas de la retina con normalidad de perfil foveal y preservación de las células ganglionares de la retina maculares (Fig. $1 \mathrm{C}$ y D).

A los 6 meses, la AV era de no percepción de luz en el OD y de cuenta dedos en el OS. Se había resuelto el edema peripapilar en el fondo de ojo con tendencia a la atrofia óptica (Fig. 2 A-D), y la OCT demostró una disminución generalizada y progresiva de la capa de fibras nerviosas de la retina (en especial en el sector temporal del disco óptico). Inicialmente hubo un respeto de las células ganglionares de la retina del área macular, pero más adelante también evolucionaron a la atrofia (Fig. 2 E y F).

\section{Discusión}

La exposición a disolventes de pintura puede causar una intoxicación múltiple, ya que contienen varios tipos de disolventes orgánicos ${ }^{1,5}$. La absorción sistémica, por vía inhalatoria u oral, es muy rápida, siendo más lenta a través de la piel intacta ${ }^{3,5}$. Los individuos habituados a intoxicarse inhalando disolventes orgánicos suelen tener adicción a otras drogas de abuso. Los disolventes orgánicos con mayor potencial tóxico presentes en los disolventes de pintura son, principalmente, el metanol y el tolueno (en este orden), aunque este último suele ser el componente más abundante $(50-70 \%)^{3}$. Otros componentes, como el acetato de metilo y la propanona (acetona), son toxicológicamente menos relevantes y actúan como agentes irritantes de la piel y las mucosas, aunque en exposiciones de 

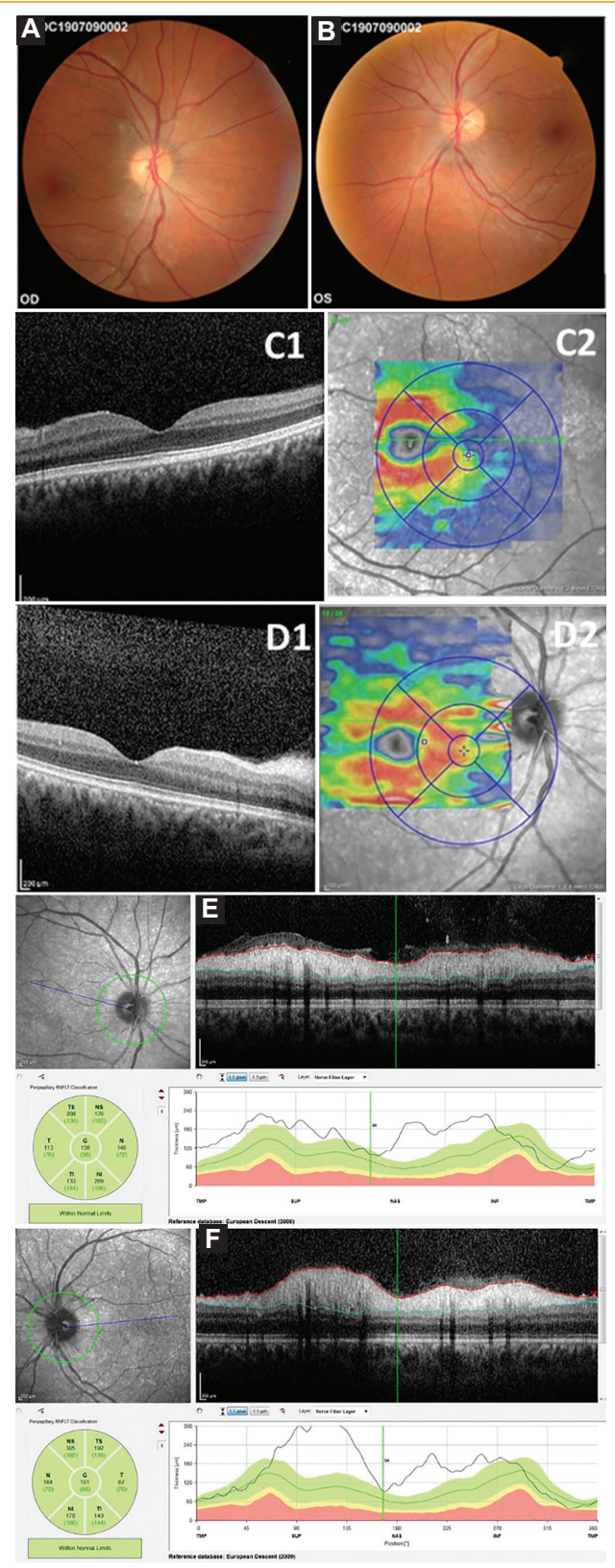

Figura 1. A: a los 3 días del inicio, imágenes de funduscopia del ojo derecho (OD) y del ojo izquierdo (OS). B: edema papilar tenue, poco congestivo y con preservación de la coloración papilar. Tomografía de coherencia óptica (OCT) macular del OD (C1) y del OS (C2): perfil foveal conservado, engrosamiento del haz papilomacular, de la capa de fibras nerviosas de la retina (CFNR) peripapilar, más evidentes en el OD, y aumento de hiperreflectividad de la capa plexiforme externa, por visibilidad de la capa de fibras de Henle debido a un descentramiento en la adquisicion de la imagen. Análisis de células ganglionares maculares del OD (D1) y del OS (D2) sin alteraciones. El centrado de las imágenes es defectuoso por falta de fijación foveal. La OCT de CFNR peripapilar del OD (E) y del OS (F) muestra un aumento generalizado del espesor, menos acusado en los sectores temporales.

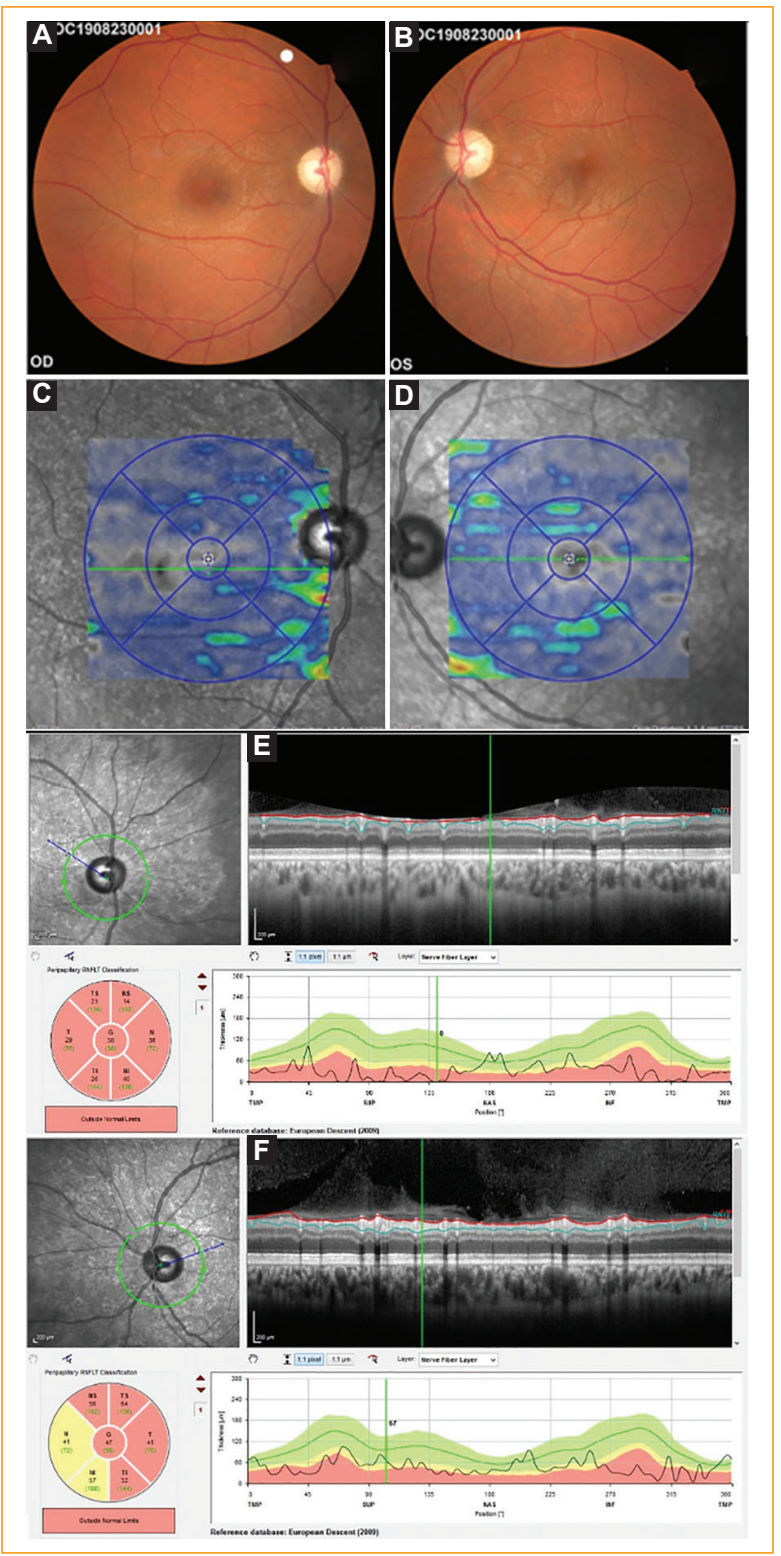

Figura 2. A los 6 meses de evolución. A: las imágenes de funduscopia del ojo derecho (OD) y del ojo izquierdo (OS). B: muestran la resolución del edema papilar, con aumento de la excavación y la palidez del anillo neurorretiniano. C: el análisis de células ganglionares maculares del OD y D: del OS demuestra una atrofia generalizada, y E: en la tomografía de coherencia óptica de la capa de fibras nerviosas de la retina peripapilar del OD y F: del OS se observa una atrofia más acusada en el OD.

mayor intensidad podrían ejercer un efecto depresor del sistema nervioso central (SNC) ${ }^{1}$.

El tolueno es un líquido incoloro, muy volátil con un olor característico («dulce»), muy lipófilo y con una gran afinidad por el SNC ${ }^{1,6}$, pero también se distribuye por el 
tejido adiposo, la médula ósea, las glándulas adrenales, el hígado y el riñón ${ }^{3,4}$. Al ser inhalado como sustancia de abuso, se observa una rápida aparición (20-30 minutos) de efectos psicoactivos, como euforia, mareo, alucinaciones y alteraciones del habla, y después incoordinación, letargia, estupor y coma ${ }^{3,5}$. En las intoxicaciones agudas y graves son característicos los desequilibrios hidroelectrolíticos, como la acidosis metabólica hiperclorémica con anión gap normal (acidosis renal distal tipo I) y la hipopotasemia, que se manifiesta por debilidad muscular y arritmias cardiacas que pueden llegar a ser mortales ${ }^{1,2,4,6}$. El tolueno se puede excretar directamente (en orina o en aire pulmonar), pero la mayor parte se metaboliza en el hígado a ácido benzoico, el cual se conjuga con la glicina produciendo ácido hipúrico, que se excreta en orina ${ }^{3}$. Tras una exposición aguda, la vida media en sangre es inferior a 24 horas. El tratamiento consiste en medidas de soporte, lavado gástrico si procede, monitorización electrocardiográfica y corrección de las alteraciones hidroelectrolíticas, especialmente de la hipopotasemia ${ }^{3,4,6}$.

El metanol es un alcohol incoloro con un olor similar al del etanol, que aparte de ser un disolvente de uso industrial es ilícitamente utilizado como adulterante de licores alcohólicos ${ }^{7-9}$. Su ingesta, aun en cantidades pequeñas ${ }^{7,9}$, puede resultar extremadamente tóxica, produciendo ceguera bilateral, alteraciones metabólicas, disfunciones neurológicas graves e incluso la muerte? Se ha estimado que la mortalidad por su intoxicación a pesar del tratamiento adecuado es del $30-40 \%{ }^{7}$, y que las alteraciones visuales permanentes podrían estar presentes en un $20-40 \%$ de los supervivientes, con una importante merma de la calidad de vida ${ }^{7,8}$. En nuestro medio, la causa más frecuente de intoxicación es deliberada como gesto autolítico, aunque hay que descartar exposición ambiental (por vía inhalatoria o transdérmica) en cuadros subagudos. La acción tóxica del metanol no se debe a su acción directa, sino al acúmulo de metabolitos como el formaldehído y el ácido fórmico derivados de su oxidación en el hígado 7 , por lo que habría una latencia en sus efectos ${ }^{8}$. Inicialmente, el gap osmolar puede estar elevado por el acúmulo de metanol, que tiene un bajo peso molecular. El metanol es metabolizado por la alcohol deshidrogenasa hepática para generar formaldehído, que a su vez se transforma en ácido fórmico por acción de la aldehído deshidrogenasa ${ }^{8,10}$. El ácido fórmico, junto con la acumulación de lactato derivado del metabolismo anaerobio, producen una acidosis metabólica con aumento del anión gap, que tendría correlación con el grado de daño visual. Se produce una hipoxia histotóxica por inhibición de la citocromo C oxidasa mitocondrial, que llevaría a la depleción de ATP, una peroxidación de los lípidos de membrana y la muerte de las células ganglionares de la retina ${ }^{10}$. Los nervios ópticos, la retina, los ganglios de la base (putamen y núcleo caudado) y la sustancia blanca subcortical serían los tejidos más susceptibles, por sus altos índices metabólicos. En los nervios ópticos habría también una desmielinización aguda en su porción retrolaminar, que contribuiría de manera indirecta a la degeneración axonal ${ }^{10}$. Esto podría explicar, en nuestro paciente, la preservación inicial del espesor de la capa de células ganglionares de la retina, que al cabo de mes y medio se adelgazó completamente, por degeneración retrógrada e interrupción de las conexiones axón-mielina ${ }^{8,10}$.

Los primeros síntomas de la intoxicación por metanol son inespecíficos: náuseas, vómitos, cefalea, molestias abdominales y deterioro del nivel de consciencia?.

Los síntomas visuales se presentan tras un periodo de latencia de 6 a 48 horas desde la exposición ${ }^{10}$ : visión borrosa, visión de "copos de nieve», fotofobia, cambios en la percepción del color, retracciones del campo visual, escotomas centrales o centrocecales y pérdida grave de visión? ${ }^{7}$ Característicamente se afectan las pupilas, que aparecen en midriasis arrefléxica?

El diagnóstico se basa en el antecedente de ingestión de metanol, ya sea accidental o intencionada, pero también se han descrito casos por exposición crónica por vía inhalatoria y transdérmica. En cualquier caso, se debe sospechar en todo varón joven o de mediana edad sin morbilidad previa, salvo conductas adictivas a sustancias de abuso, que presente una neuropatía óptica bilateral de instauración aguda acompañada de acidosis metabólica con anión gap aumentado, encefalopatía y dolor abdominal ${ }^{8}$. En el caso de la intoxicación aguda por tolueno aparecerían los síntomas sugestivos de toxicidad en minutos: dolor abdominal, embriaguez y debilidad muscular con riesgo de arritmias cardiacas por hipopotasemia. Se han reportado casos de neuropatías ópticas bilaterales, que se presentan como atrofia óptica, producidas por exposiciones muy prolongadas ${ }^{4,6}$, pero no por intoxicaciones agudas $^{1,6}$.

Para confirmar el diagnóstico es útil la determinación de la concentración sérica de metanol (no siempre disponible), realizar un electrocardiograma y verificar la existencia de una acidosis metabólica con una brecha osmolar inicialmente elevada que se normaliza conforme aumenta el anión gap ${ }^{7}$. En el caso del tolueno, se produce una acidosis metabólica 
caracterizada por anión gap normal, hipercloremia e hipopotasemia, que se debe a su acción toxica en el túbulo renal distal que mermaría su capacidad de excretar $\mathrm{H}^{+}$y retener $\mathrm{K}^{+3}$. En nuestro caso, fue el acúmulo de ácido fórmico (derivado del catabolismo del metanol) y de lactato (derivado del metabolismo anaeróbico) el principal determinante de la acidosis (con anión gap elevado), aunque también se produjo hipopotasemia como consecuencia de la pérdida en el túbulo renal.

Los hallazgos de fondo de ojo en la fase aguda pueden ser en forma de papila hiperémica con edema de la capa de fibras peripapilares, edema de papila y neurorretinitis, aunque también es posible observar un aspecto normal, sugestivo de afectación retrobulbar. La palidez del disco y el estrechamiento de los vasos sanguíneos se instaurarían en el plazo de 6 a 12 semanas, y habitualmente la palidez precede al estrechamiento de los vasos. También es característica en esta fase de atrofia un aumento de la excavación papi$\operatorname{lar}^{10}$, así como un progresivo adelgazamiento de la capa de fibras peripapilares, sobre todo temporales, valorado mediante OCT; ambos hallazgos estaban presentes en nuestro paciente. Se han descrito otras alteraciones retinianas, como la aparición de microquistes en la capa nuclear interna ${ }^{11}$, ausentes en nuestro caso.

El tratamiento de la intoxicación por metanol consiste en la instauración de medidas de alcalinización (bicarbonato intravenoso) y hemodiálisis (en los casos más graves) para la corrección del pH ácido7,8. Además de tratar la acidosis metabólica y favorecer la eliminación de los metabolitos derivados, se debe inhibir el metabolismo del metanol. El etanol y el fomepizol (no depresor del SNC) son antídotos específicos, ya que bloquean la degradación del metanol a formaldehído al tener una alta afinidad por la alcohol deshidrogenasa. La administración intravenosa de suplementos vitamínicos, como ácido folínico, tiamina, piridoxina y metilcobalamina, puede ser beneficiosa al favorecer el metabolismo del ácido fórmico ${ }^{9}$. En nuestro caso, además, fue sumamente importante la monitorización electrocardiográfica y la corrección de la hipopotasemia. El uso de corticoides a altas dosis o de eritropoyetina podría ser beneficioso, pero no hay evidencia clínica suficiente ${ }^{9}$.

Se puede iniciar la recuperación visual a partir de las 2-3 semanas tras la exposición al metanol, aunque en un $25-40 \%$ de los casos quedan perturbaciones de la $\mathrm{AV}$, de la percepción del color o del campo visual $^{10}$.
Se ha hallado que el principal factor pronóstico de la AV final (y de la mortalidad) es la gravedad de la acidez metabólica inicial ${ }^{7}$, en comparación con otros factores estudiados, como la midriasis, la edad y la precocidad del tratamiento ${ }^{7,9}$.

\section{Conclusiones}

En las intoxicaciones agudas por la combinación de metanol y tolueno se producen síntomas inespecíficos y comunes, como dolor abdominal, encefalopatía y acidosis metabólica, de aparición más rápida con el tolueno. A estos se sumarían los específicos de cada sustancia: la hipopotasemia característica del tolueno y la neuropatía óptica aguda bilateral de carácter grave y progresivo del metanol. La integridad de las células ganglionares de la retina en la exploración inicial en nuestro paciente no tuvo valor pronóstico, pues evolucionó a atrofia óptica total.

\section{Financiamiento}

Este trabajo no ha recibido ningún financiamiento para su realización.

\section{Conflicto de intereses}

Ninguno de los autores de este manuscrito tiene conflictos de intereses.

\section{Responsabilidades éticas}

Protección de personas y animales. Los autores declaran que para esta investigación no se han realizado experimentos en seres humanos ni en animales.

Confidencialidad de los datos. Los autores declaran que han seguido los protocolos de su centro de trabajo sobre la publicación de datos de pacientes.

Derecho a la privacidad y consentimiento informado. Los autores han obtenido el consentimiento informado de los pacientes y/o sujetos referidos en el artículo. Este documento obra en poder del autor de correspondencia.

\section{Bibliografía}

\footnotetext{
1. Joshi DR, Adhikari N. An overview on common organic solvents and their toxicity. JPRI. 2019;28:1-18.

2. Allam HK, Soliman S, Wasfy T, Ghoneim A, Serag Y, Sembajwe G. The neuro-ophthalmological effects related to long-term occupational exposure to organic solvents in painters. Toxicol Ind Health. 2018; $34: 91-8$.
} 
3. Camara-Lemarroy CR, Rodríguez-Gutiérrez R, Monreal-Robles R, González-González JG. Acute toluene intoxication - clinical presentation, management and prognosis: a prospective observational study. BMC Emerg Med. 2015;15:19.

4. Twardowschy CA, Teive HA, Siquineli F, Fernandes AF, Búrigo IP, Carvalho-Neto $A$, et al. Optic neuritis due to solvent abuse. Arq Neuropsiquiatr. 2008;66:108-10.

5. Howard MO, Bowen SE, Garland EL, Perron BE, Vaughn MG. Inhalant use and inhalant use disorders in the United States. Addict Sci Clin Pract. 2011;6:18-31.

6. Gupta SR, Palmer CA, Curé JK, Balos LL, Lincoff NS, Kline LB. Toluene optic neurotoxicity: magnetic resonance imaging and pathologic features. Hum Pathol. 2011;42:295-8
7. Sharma R, Marasini S, Sharma AK, Shrestha JK, Nepal BP. Methanol poisoning: ocular and neurological manifestations. Optom Vis Sci. 2012;89:178-82.

8. Galvez-Ruiz A, Elkhamary SM, Asghar N, Bosley TM. Visual and neurologic sequelae of methanol poisoning in Saudi Arabia. Saudi Med J. 2015;36:568-74

9. Desai T, Sudhalkar A, Vyas U, Khamar B. Methanol poisoning: predictors of visual outcomes. JAMA Ophthalmol. 2013;131:358-64.

10. Nurieva O, Hubacek JA, Urban $P$, Hlusicka J, Diblik $P$, Kuthan $P$, et al. Clinical and genetic determinants of chronic visual pathway changes after methanol-induced optic neuropathy: four-year follow-up study. Clin Toxicol. 2019:57:387-97.

11. Klein KA, Warren AK, Baulman CR, Hedges III TR. Optical tomography findings in methanol toxicity. Int J Retin Vitr. 2017;3:36. 